\title{
La Impulsividad y su Asociación con la Inclinación de la Frente
}

\author{
Impulsivity and its Association with the Slant of the Forehead
}

David Guerrero",**; Cristina Gabarre-Armengol**; José Blas Navarro*** \& Julián Gabarre-Mir***

GUERRERO, D.; GABARRE-ARMENGOL, C.; NAVARRO, J. B. \& GABARRE-MIR, J. La impulsividad y su asociación con la inclinación de la frente. Int. J. Morphol., 34(3):990-996, 2016.

RESUMEN: Avances en embriología, genética o neuroimagen, están ayudando a discernir posibles correlaciones entre estructuras del rostro, cerebro y personalidad. Una conducta que ha sido objeto de numerosas investigaciones es la impulsiva, de la que han surgido distintas conceptualizaciones con cierta controversia etiológica. En el presente trabajo estudiamos si el rostro refleja de manera objetiva el nivel de impulsividad. Para ello, se utilizó en población adulta $(\mathrm{N}=33)$, la dimensión Búsqueda de Novedad del Inventario de Temperamento y Carácter-Revisado de Cloninger, la Escala de Impulsividad de Barratt (BIS-11) y la Escala de Impulsividad Estado (EIE). En el rostro se midió el grado de inclinación de la frente hacia atrás, resultando una asociación positiva con las 3 puntuaciones psicométricas. Tres jueces evaluaron visualmente el nivel de inclinación de la frente. Se obtuvo un alto acuerdo entre ellos, así como una alta correlación con la medida objetiva de inclinación. Aunque la muestra es reducida, los datos ofrecen una aproximación al objetivo de estudio.

PALABRAS CLAVE: Impulsividad; Rostro; Forma; Craneofacial; Córtex prefrontal.

\section{INTRODUCCIÓN}

La historia de la Psicología refleja el interés por las formas y estructuras del ser humano, siendo cráneo y rostro las más estudiadas para discernir la personalidad e inteligencia. Aunque parecía existir una evidencia emergente entre morfología y comportamiento (Gordon et al., 2008), la realidad actual es que existen pocos estudios que asocien la estructura corporal con aspectos de personalidad. Por ejemplo, se ha visto como la altura o la elevada flexibilidad articular se asocia con niveles de ansiedad (Baeza Velasco \& Bulbena, 2009) o lo encontrado en Gabarre (2010) donde a nivel craneofacial las diferencias métricas entre la anchura del arco cigomático comparadas con la anchura mandibular, proporcionaban información sobre expresividad emocional y adhesión grupal.

Una conducta que es objeto de numerosas investigaciones es la impulsiva de la que han surgido distintas conceptualizaciones con cierta controversia etiológica. Recientes investigaciones apuntan a que áreas prefrontales (cingulado anterior / zona ventromedial) ejercen un control inhibitorio del impulso procedente del núcleo estriado (Fineberg et al., 2014). En este sentido, en población sana, (Boes et al., 2009; Matsuo, 2009; Schilling et al., 2013b) han visto asociaciones inversas entre el volumen de áreas prefrontales e impulsividad. La misma asociación se ha encontrado en pacientes dependientes de alcohol (Tornese et al., 2013) o con trastorno límite de personalidad (O'Neill et al., 2013), cuyos rasgos nucleares de estos trastornos son la conducta impulsiva.

Respecto a la estructura craneofacial, DonovanLepore et al., (2006) apuntan que el cráneo deriva de las células de la cresta neural y del mesodermo e influencia el desarrollo de las estructuras del cerebro. En la misma línea, Kjaer (1995) habla del desarrollo interconectado del cerebro-rostro o Diewert \& Lozanoff (1993) que concluyen que "el rostro está esencialmente aguantado por el cerebro creciente, que sufre amplios cambios en el desarrollo de su morfología". Por lo visto anteriormente, si se tiene en cuenta que el crecimiento del encéfalo puede estar condicionado

\footnotetext{
* Departament of Psychiatry and Legal Medicine. PhD Programme in Psychiatry. Universidad Autónoma de Barcelona. Spain

** Institute of Craniofacial Psychology. Barcelona. Spain.

**** Department of Psychobiology and Methodology of the Behavioural Sciencies. Universidad Autónoma de Barcelona. Spain.
} 
por el cráneo que le da soporte y protección, una estructura más inclinada hacia atrás de la zona frontal de la bóveda craneal (frente) podría albergar un menor volumen del área prefrontal-frontal lo que podría traducirse en un mayor déficit inhibitorio de la conducta y en consecuencia en una mayor impulsividad. Así, ya que existen diferencias antropométricas en la inclinación de la frente vista de perfil, el objetivo del presente trabajo fue estudiar la influencia de los niveles de inclinación de la frente sobre la impulsividad, y observar si una mayor inclinación se asocia a puntuaciones más altas en impulsividad.

\section{MATERIAL Y MÉTODO}

Participaron 33 adultos seleccionados de población general y con diferentes niveles educativos (primarios: 6.1 $\%$, secundarios: $57.6 \%$, superiores: $36.4 \%$ ) (ver Tabla I para descripción de la muestra). Para obtener la muestra se empleó un muestreo no probabilístico. Se propuso participar a 76 personas seleccionadas al azar de entre aquellos que habían coincidido con el investigador principal en diferentes centros de formación sobre psicología, en la ciudad de Barcelona y provincia. Todos los participantes firmaron un consentimiento informado para realizar la evaluación y autorizaron el uso de los datos para investigación, de acuerdo con la declaración de derechos de Helsinki.

Se establecieron como criterios de exclusión: prescripción de algún psicofármaco o diagnóstico de trastorno mental y/o neurológico severo.

A cada participante se le tomó una fotografía del perfil izquierdo con una cámara digital réflex, marca Canon, modelo EOS 1100 D EF-S 18-55, adaptada a un trípode de altura regulable. Se fijó una distancia de 1.4 metros y una obertura focal de $50 \mathrm{~mm}$. Sentados en un taburete de altura regulable se efectuaron las fotografías con la cabeza recta, erguida y mirando al frente. El fondo era gris oscuro y se utilizó luz artificial.
Las fotografías fueron ajustadas y maquetadas para su impresión en un centro de fotografía profesional en formato DIN-A4. Para mantener las proporciones y el encuadre se dejó un margen de $3 \mathrm{~cm}$ (parte derecha y superior) y $2 \mathrm{~cm}$ (parte izquierda). En soporte fotográfico, los grados de inclinación del ángulo de la frente se midieron con un trasportador de ángulos semicircular marca Staedtler 568 de $10 \mathrm{~cm}$ de regla. Para ello, se tomaron de la metodología creada por Farkas (1994) 2 puntos antropométricos de referencia: Trichion (tr), punto en la línea media de la frente que se ubica en el nacimiento del pelo, y Glabella (g), punto de la línea media más prominente entre las dos cejas. Se trazó una línea recta vertical ascendente desde el punto (g), hasta converger en ángulo recto con la línea trazada en horizontal desde el punto (tr) (Fig. 1).

Por otro lado, durante cinco horas, se entrenó a 3 jueces observadores (un doctor en psicología, una licenciada en psicología y una técnica superior de dibujo) para efectuar una medición visual del nivel de inclinación de la frente hacia atrás (NIF). Las evaluaciones se hicieron por separado en una sala con proyector habilitada por el investigador y la medida para la evaluación quedó sujeta a la siguiente clasificación: 1-Nulo, 2- Bajo, 3- Medio, 4Medio-alto y 5- Alto.

\section{Pruebas psicométricas:}

a) Inventario de Temperamento y Carácter-Revisado de Cloninger (TCI-R): desarrollado por Cloninger et. al, (1994) y adaptado a población española por GutiérrezZotes et al. (2004). Dispone de 240 ítems respuesta tipo Likert. Evalúa 7 dimensiones básicas de la personalidad y 29 subdimensiones que forman parte de su Teoría Biopsicosocial. En temperamento se encuentran 4 dimensiones (Evitación del Riesgo -ER-, Búsqueda de Novedad -BN-, Dependencia en la Recompensa -DR- y Persistencia -P-). En carácter se incluyen 3 dimensiones (Autodirección-A-, Cooperación -C-y Autotrascendencia -T-). Para el presente estudio se analizaron BN y ER. Las

Tabla I. Descriptivos de la muestra, pruebas psicométricas y GIF.

\begin{tabular}{lcccccc}
\hline & $\mathrm{N}$ & Media & DE & Mínimo & Máximc & Mediana \\
\hline Edad & 33 & 35.61 & 9.98 & 22 & 62 & 33 \\
Grados Inclinación Frente (GIF) & 33 & 11.03 & 6.25 & 2 & 26 & 9 \\
Hombres & 16 & 12.56 & 7.87 & 2 & 26 & 16 \\
Mujeres & $(48.5 \%)$ & 9.59 & 3.92 & 4 & 16 & 9 \\
& 17 & & & & & \\
TCI-R: Búsqueda de Novedad (BN) & $(51.5 \%)$ & & & & & \\
TCI-R: Evitación del Riesgo (ER) & 33 & 59.61 & 31.93 & 2 & 99 & 60 \\
BIS 11 Total & 33 & 41.58 & 30.29 & 1 & 98 & 40 \\
EIE Total & 33 & 41.24 & 16.02 & 17 & 81 & 38 \\
& 33 & 11.91 & 9.03 & 2 & 35 & 10 \\
\hline
\end{tabular}




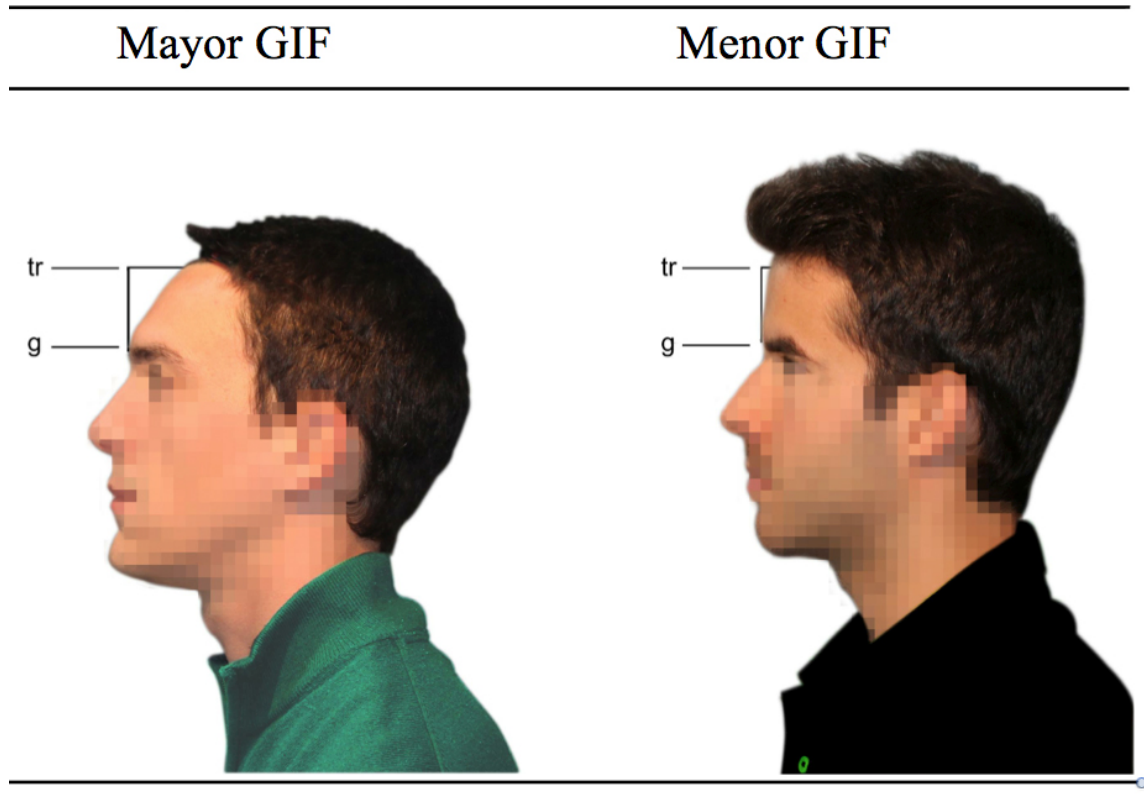

Fig. 1. Ejemplo de la medición de los grados del ángulo de inclinación de la frente (GIF). Puntos antropométricos de referencia: Trichion (tr), punto en la línea media de la frente, que se ubica en el nacimiento del pelo y Glabella (g), punto de la línea media más prominente entre las dos cejas.

propiedades psicométricas son adecuadas, con un alfa de Cronbach de 0,77 para BN y de 0,84 para ER (GutiérrezZotes et al., 2004).

b) Escala de Impulsividad de Barratt (BIS-11) (Patton, 1995): adaptado a población española por Oquendo et al. (2011), mide la impulsividad entendida como rasgo de personalidad. Consta de 30 ítems que se puntúan en una escala tipo Likert de 0 (raramente o nunca), 1 (ocasionalmente), 3 (a menudo) y 4 (siempre o casi siempre). La corrección se efectúa en los términos de a mayor puntuación mayor impulsividad. La versión española muestra unas adecuadas propiedades psicométricas, con una consistencia interna alfa de 0,75 y una fiabilidad test-retest tras 2 meses que proporciona un Coeficiente de Correlación Intraclase (CCI) de 0,89.

c) Escala de Impulsividad Estado (EIE): escala clínica, validada en población española por Iribarren et al. (2011), que permite medir la conducta impulsiva como estado. Se presentan 20 ítems, agrupados en 3 dimensiones: gratificación, automatismo y atencional. Con respuesta tipo Likert de 4 opciones que indican que a puntuaciones más altas mayor impulsividad. Se utilizó como medida la puntuación total. Ha mostrado una elevada fiabilidad, con un alfa de 0,884 .

En el análisis estadístico, se estudió mediante el Coeficiente de Correlación Intraclase (CCI) la concordancia de la medida visual, nivel de inclinación de la frente hacia atrás (NIF), de los 3 jueces. Posteriormente, se efectúo una única medida promedio de los 3 jueces. Con la medida visual promedio de NIF, se calculó el coeficiente de correlación de Pearson con la medida objetiva de grados de inclinación de la frente hacia atrás (GIF). A través de una regresión lineal múltiple, se analizó la influencia de GIF sobre las puntuaciones de impulsividad en los test psicométricos y se estudió la posible existencia de interacción y confusión producida por sexo y edad. Siguiendo la propuesta de Maldonado \& Greenland (1993), se mantuvieron las variables de ajuste en el modelo cuando el cambio entre el efecto bruto y el ajustado era mayor al $10 \%$. Los datos se analizaron con el paquete estadístico SPSS versión 19.

\section{RESULTADOS}

El nivel de concordancia de los 3 jueces para la evaluación visual NIF proporcionó un CCI= 0.961 (IC $95 \%$ : 0,929 a 0,979). Dado el alto grado de acuerdo interjueces, se obtuvo la medida promedio del nivel de inclinación de la frente (NIF). La correlación entre la medida promedio y el grado de inclinación de la frente (GIF) fue muy alta: NIF $(\mathrm{r}=0,88, \mathrm{p}<0,0005)$ lo que muestra una altísima asociación entre la evaluación visual y la antropométrica.

La Tabla II muestra la influencia de GIF, sobre las dimensiones BN y ER del TCI-R y sobre las puntuaciones obtenidas en BIS-11 y EIE. 
Tabla II. Efecto de GIF sobre BN, ER, BIS-11 y EIE.

\begin{tabular}{lcrrr}
\hline & $\mathrm{b}$ & $\mathrm{t}$ & \multicolumn{1}{c}{$\mathrm{p}$} & \multicolumn{1}{c}{ IC 95\% } \\
\hline TCI-R: Búsqueda de Novedad (BN)* & 2,180 & 2,41 & 0,025 & 0,330 a 4,030 \\
TCI-R: Evitación del Riesgo (ER)* & 0,205 & 0,20 & 0,843 & $-1,886$ a 2,295 \\
BIS 11 Total & 1,615 & 4,51 & $<0,0005$ & 0,885 a 2,345 \\
EIE Total & 0,762 & 3,45 & 0,002 & 0,312 a 1.212 \\
\hline
\end{tabular}

*Ajustado por sexo y edad

En la Figura 2 se pueden ver conjuntamente los gráficos de dispersión entre GIF-BN y GIF-ER. En la dimensión BN, el estudio de interacción por sexo y edad obtuvo unos datos no significativos $(\mathrm{p}=0,101)$, aunque ambas variables demográficas sí fueron introducidas como medidas de ajuste estadístico. Se concluye que por cada grado que aumenta la inclinación de la frente, la puntuación en BN aumentará 2,180 puntos (IC $95 \%$ : 0,330 a 4,030), con un grado de significación $\mathrm{p}=0,025)$. En la dimensión $\mathrm{ER}$, la interacción con sexo y edad tampoco obtuvo significación $(\mathrm{p}=0,081)$. Ajustado por sexo y edad se halló que por cada grado que aumenta la inclinación de la frente, ER aumentará no significativamente 0,205 puntos (IC $95 \%$ : $-1,886$ a $2,295 ; \mathrm{p}=0,843)$.
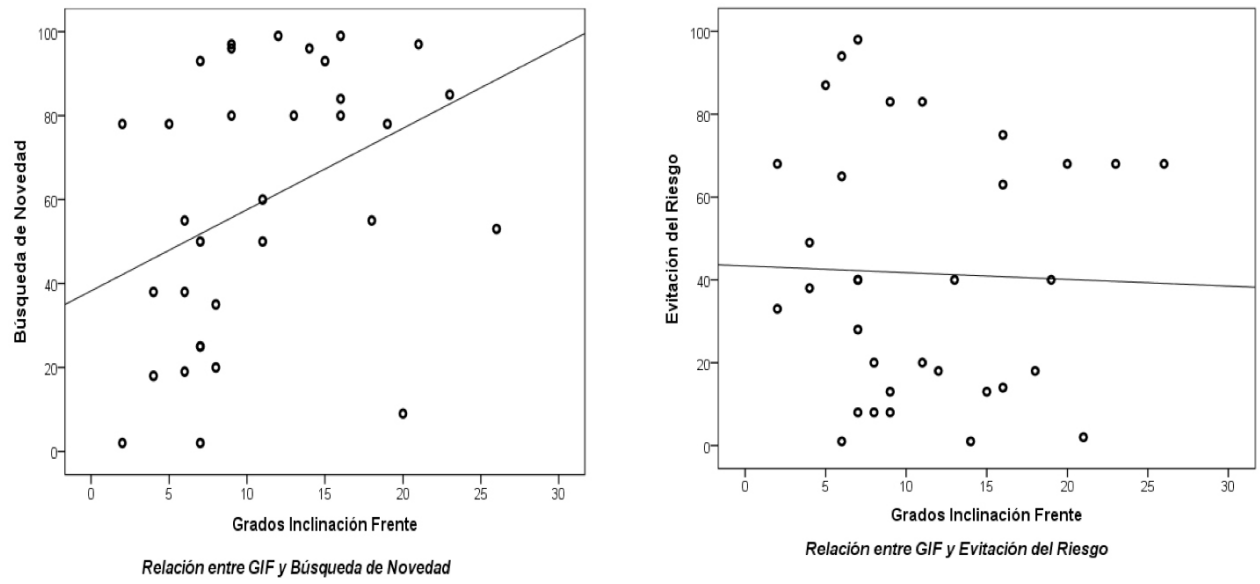

Fig. 2. Comparativa de los gráficos de dispersión entre grados de inclinación de la frente (GIF) y las dimensiones de TCI-R (Búsqueda de Novedad y Evitación del Riesgo).

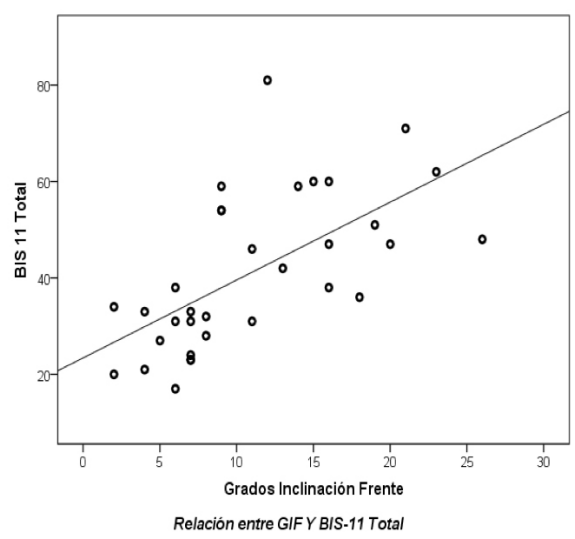

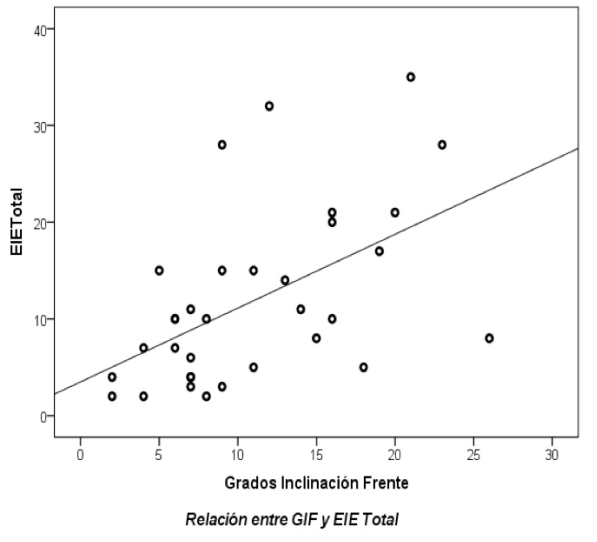

En la Figura 3 pueden verse los gráficos de dispersión entre GIF y Escala Impulsividad de Barratt (BIS-11) así como entre GIF y Escala de Impulsividad Estado (EIE).

En BIS-11, la interacción por sexo y edad resultó no significativa ( $\mathrm{p}=$ $0,453)$ y no fue necesario el ajuste ni por sexo ni por edad. Se obtuvo que por cada grado que aumenta la inclinación de la frente el BIS-11 total aumentará significativamente 1.615 puntos (IC $95 \%$ : 0,885 a $2,345 ; \mathrm{p}<0,0005)$.

Finalmente para EIE, tampoco se obtiene interacción ni con sexo ni con edad $(\mathrm{p}=0,394)$, ni se considera necesario el ajuste por estas variables. Se concluye que por cada grado que aumenta la inclinación de la frente el EIE total aumentará significativamente 0,762 puntos (IC95 \%: 0,312 a 1,212; $\mathrm{p}=0,002)$.

Fig.3. Gráficos de dispersión entre GIF-BIS-11 y GIF-EIE. 


\section{DISCUSIÓN}

En primer término, se observó una alta fiabilidad interjueces en la medida visual y una alta correlación de la medida promedio de la evaluación visual con los grados de inclinación de la frente. De ello se deduce que la evaluación visual del grado de inclinación de la frente puede ser suficiente y evitar una instrumentación más compleja para medidas antropométricas.

En cuanto a las medidas psicométricas de la impulsividad, la asociación más baja se da en la dimensión Búsqueda de Novedad. Esta dimensión, no sólo está constituida por la evaluación de la impulsividad, sino que incluye facetas como actividad exploratoria, extravagancia y planificación (Gutiérrez-Zotes et al.,), lo que podría explicar esta más baja asociación. En comparación, la dimensión Evitación del Riesgo describe a los sujetos con mayor inhibición conductual, aburrimiento y pesimismo, considerándose más ansiosos y menos impulsivos, lo que va en consonancia con nuestros resultados y con los estudios que describen que sujetos sanos con un patrón conductual de Búsqueda de Novedad obtienen puntuaciones opuestas en Evitación del Riesgo (Laricchiuta et al., 2014).

Los marcos teóricos que definen la impulsividad incluyen conductas de búsqueda activa de recompensas, evitación de la frustración, actuaciones rápidas y una baja previsión de las consecuencias (Squillace et al.). Uno de los test más utilizados para evaluar el rasgo de impulsividad es el BIS11, que mide las definiciones anteriores (Squillace et al., 2011). Así, dado la mayor asociación de GIF con BIS-11 que incluye diferentes facetas del constructo, se podría sugerir que una alta inclinación de la frente constituye un fenotipo conductual e informa de un rasgo estable de impulsividad sin entrar en consideraciones clínicas, alejadas de este estudio. La evaluación de la impulsividad como estado a través de la escala EIE, muestra una asociación positiva menor que la anterior, siendo los datos esperables, ya que hay que considerar que sujetos con un rasgo bajo en impulsividad pueden actuar de manera puntual en la dirección opuesta o viceversa, movidos por situaciones ambientales concretas en el momento de la evaluación (Iribarren).

Por otro lado, en un novedoso estudio, se encontró que la forma craneofacial puede informar de facetas de personalidad (Gabarre), lo que soporta, por lo menos en parte, lo descrito por Sisodiya et al. (2008) que comentan que "recientes avances en la comprensión del cerebro humano a través de entidades íntimamente vinculadas, como el rostro (...) pueden facilitar información directa acerca del cerebro". Además, si tenemos en cuenta los datos encontrados en el presente estudio, la función inhibitoria de la corteza prefrontal y así como su correspondencia con la zona frontal de la bóveda craneal, sería factible investigar la inclinación de esta zona craneal y su asociación con el volumen prefrontal e impulsividad. Está hipótesis puede sostenerse con explicaciones como la de DeMeyer (1975) que habla de que "el subyacente cerebro y el recubriente rostro están complicadamente entrelazados" o con estudios de desarrollo craneal donde Kjaer (1995) expone que "el rostro, el esqueleto craneofacial y el sistema nervioso central y periférico tienen un desarrollo interconectado".

En cuanto a las limitaciones del presente estudio, hay que considerar el tamaño reducido de la muestra, y en consecuencia la generalización de nuestros resultados. Por otro lado, existe la dificultad de encontrar un instrumento de medición antropométrica directa de la inclinación de la frente. Respecto a las mediciones psicométricas, este trabajo se ha centrado exclusivamente en dos dimensiones de temperamento del TCI-R, y se ha utilizado como equivalente a impulsividad, la dimensión BN y una segunda de ER, como dimensión comparativa y opuesta que puede reflejar ansiedad. En consecuencia, no se ha profundizado sobre distintas subdimensiones que definen la faceta de BN, así como en otras dimensiones de temperamento y carácter definidas en este test, por lo que en futuros estudios sería interesante estudiar los grados de inclinación de la frente con las variables excluidas así como con las distintas subescalas de BIS11 y EIE que no han sido analizadas.

Finalmente, hasta donde llega nuestro conocimiento y tomados los datos con cautela, éste trabajo es de los primeros que puede acercarse a describir que una estructura tipológica craneofacial puede sugerir niveles de impulsividad, lo que puede ayudar, en sí mismo, en la predicción de este rasgo de personalidad en la población.

AGRADECIMIENTOS: A las personas que de forma desinteresada y voluntaria han participado en este estudio.

GUERRERO, D.; GABARRE-ARMENGOL, C.; BLAS NAVARRO, J. \& GABARRE-MIR, J. Impulsivity and its association with the slant of the forehead. Int. J. Morphol., 34(3):990-996, 2016.

SUMMARY: Advances in embryology, genetics and neuroimaging are helping to discern possible correlations between facial structures, brain and personality. A behavior that has been the subject of much research is impulsiveness, where different 
conceptualizations have arisen with some etiological controversy. Objectifying the possibility of different levels of impulsivity in the face is studied. For an adult sample $(\mathrm{N}=33)$, the Novelty Seeking dimension of Temperament and Character-Revised by Cloninger, the Barratt Impulsiveness Scale (BIS-11) and Impulsiveness Scale State (EIE) were used. In the forehead the degree of inclination front to back was measured, resulting in a positive association with the 3 psychometric scores. Three judges evaluated the level of inclination of the forehead, resulting in a high reliability and a high correlation with the objective measurement of inclination. Although the sample is small, the data provides an objective approach to the study of the variable analyzed.

\section{Prefrontal cortex.}

KEY WORDS: Impulsivity; Face; Shape; Craniofacial;

\section{REFERENCIAS BIBLIOGRÁFICAS}

Baeza Velasco, C. \& Bulbena, A. Ansiedad social y alteración de colágeno en personas de gran estatura. Cuad. Med. Psicosom. Psiquiatr. Enlace, (89-90):40-6, 2009.

Boes, A. D.; Bechara, A.; Tranel, D.; Anderson, S. W.; Richman, L. \& Nopoulos, P. Right ventromedial prefrontal cortex: a neuroanatomical correlate of impulse control in boys. Soc. Cogn. Affect. Neurosci., 4(1):1-9, 2009.

Cloninger, R. C.; Pryzbeck, T. R.; Svrakic, D. M. \& Wetzel, R. D. The Temperament and Character Inventory. A Guide to its Development and Use: Amerikaanse Handleiding. St. Louis, Center for Psychobiology of Personality, Washington University, 1994.

DeMyer, W. Median facial malformations and their implications for brain malformations. Birth Defects Orig. Artic. Ser., 11(7):155-81, 1975

Diewert, V. M. \& Lozanoff, S. A morphometric analysis of human embryonic craniofacial growth in the median plane during primary palate formation. J. Craniofac. Genet. Dev. Biol., 13(3):147-61, 1993.

Donovan-Lepore, A. M.; Jaeger, J.; Czobor, P.; Abdelmessih, S. \& Berns, S. M. Quantitative craniofacial anomalies in a racially mixed schizophrenia sample. Biol. Psychiatry, 59(4):349-53, 2006.

Farkas, L. G. Anthropometry of the Head and Face. 2nd ed. New York, Raven Press, 1994.

Fineberg, N. A.; Chamberlain, S. R.; Goudriaan, A. E.; Stein, D. J.; Vanderschuren, L. J.; Gillan, C. M.; Shekar, S.; Gorwood, P. A.; Voon, V.; Morein-Zamir, S.; Denys, D.; Sahakian, B. J.; Moeller, F. G.; Robbins, T. W. \& Potenza, M. N. New developments in human neurocognition: clinical, genetic, and brain imaging correlates of impulsivity and compulsivity. $C$. N. S. Spectr., 19(1):69-89, 2014.

Gabarre, J. Rostro y Cerebro: Dos Caras de una Misma Realidad. Tesis doctoral. Barcelona, Universitat Autónoma de Barcelona, 2010 .

Gordon Jr., G. G.; Frederick, M. J. \& Pipitone, R. N. Morphology and behavior: Phrenology revisited. Rev. Gen. Psychol., 12(3):297-304, 2008.

Gutiérrez-Zotes, J. A.; Bayón, C.; Montserrat, C.; Valero, J.; Labad Alquézar, A.; Cloninger, C. R. \& Fernández-Aranda, F. Inventario del Temperamento y el Carácter-Revisado (TCI-R). Baremación y datos normativos en una muestra de población general. Actas Esp. Psiquiatr., 32(1):8-15, 2004.

Iribarren, M. M.; Jiménez-Giménez, M.; García-de Cecilia, J. M. \& Rublo-Valladolid, G. Validación y Propiedades Psicométricas de la Escala de Impulsividad Estado (EIE ). Actas Esp. Psiquiatr., 39(1):49-60, 2011.

Kjaer, I. Human prenatal craniofacial development related to brain development under normal and pathologic conditions. Acta Odontol. Scand., 53(3):135-43, 1995.

Laricchiuta, D.; Petrosini, L.; Piras, F.; Macci, E.; Cutuli, D.; Chiapponi, C.; Cerasa, A.; Picerni, E.; Caltagirone, C.; Girardi, P.; Tamorri, S. M. \& Spalletta, G. Linking novelty seeking and harm avoidance personality traits to cerebellar volumes. Hum. Brain Mapp., 35(1):285-96, 2014.

Maldonado, G. \& Greenland, S. Simulation study of confounderselection strategies. Am. J. Epidemiol., 138(11):923-36, 1993.

Matsuo, K.; Nicoletti, M.; Nemoto, K.; Hatch, J. P.; Peluso, M. A.; Nery, F. G. \& Soares, J. C. A voxel-based morphometry study of frontal gray matter correlates of impulsivity. Hum. Brain Mapp., 30(4):1188-95, 2009.

O’Neill, A.; D’Souza, A.; Carballedo, A.; Joseph, S., Kerskens, C. \& Frodl, T. Magnetic resonance imaging in patients with borderline personality disorder: a study of volumetric abnormalities. Psychiatry Res., 213(1):1-10, 2013.

Oquendo, M. A.; Baca-García, E.; Graver, R.; Morales, M.; Montalvan, V. \& Mann, J. J. Spanish adaptation of the Barratt Impulsiveness Scale (BIS-11). Eur. J. Psychiatry, 15(3):147$55,2001$.

Patton, J. H.; Stanford, M. S. \& Barratt, E. S. Factor structure of the Barratt impulsiveness scale. J. Clin. Psychol., 51(6):76874, 1995.

Schilling, C.; Kühn, S.; Romanowski, A.; Banaschewski, T., Barbot, A.; Barker, G. J.; Brühl, R.; Büchel, C.; Charlet, K.; Conrod, P. J.; Czech, K.; Dalley, J. W.; Flor, H.; Häke, I.; Ittermann, B.; Ivanov, N.; Mann, K.; Lüdemann, K.; Martinot, J. L.; Palafox, 
C.; Paus, T.; Poline, J. B.; Reuter, J.; Rietschel, M.; Robbins, T. W.; Smolka, M. N.; Ströhle, A.; Walaszek, B.; Kathmann, N.; Schumann, G.; Heinz, A.; Garavan, H.; Gallinat, J. \& IMAGEN consortium. Common structural correlates of trait impulsiveness and perceptual reasoning in adolescence. Hum. Brain Mapp., 34(2):374-83, 2013.

Sisodiya, S. Brain structure, function, and genetics revealed by studies of the eye and face. Curr. Opin. Neurol., 21(4):404-9, 2008.

Squillace, M.; Picón Janeiro, J. \& Schmidt, V. El concepto de impulsividad y su ubicación en las teorías psicobiológicas de la personalidad. Rev. Neuropsicol. Latinoam., 3(1):8-18, 2011.

Tornese, E. B. \& Mazzoglio y Nabar, M. J. Morphometry of the corpus callosum and mammillary bodies in alcoholism using magnetic resonance. Int. J. Morphol., 31(4):1233-42, 2013.

\section{Correspondencia: \\ J. David Guerrero Apolo \\ Carrer Pau Clarís \\ $N^{0} 120,3^{\circ} 2^{a}$ C. P. 08009 \\ Barcelona \\ ESPAÑA}

Teléfono: +0034687434953

Fax: $\quad+34935811435$

Email: davidguerrero@copc.cat

Recibido: 07-04-2016

Aceptado: 18-07-2016 